OPEN ACCESS

Edited by:

Venancio Avancini Alves, Universidade de São Paulo, Brazil

Reviewed by:

Pierlorenzo Pallante,

Consiglio Nazionale Delle Ricerche

$(C N R)$, Italy

Mariangela Esther Alencar Marques, Universidade Estadual Paulista Júlio de Mesquita Filho (UNESP), Brazil

*Correspondence: Cleverson T. Soares clev.blv@terra.com.br

Specialty section: This article was submitted to

Pathology,

a section of the journa

Frontiers in Medicine

Received: 09 March 2018

Accepted: 30 August 2018

Published: 24 September 2018

Citation:

Soares CT, Fachin LRV Trombone APF, Rosa PS, Ghidella CC and Belone AFF (2018) Potential of AKR1B10 as a Biomarker and Therapeutic Target in Type 2 Leprosy

Reaction. Front. Med. 5:263. doi: 10.3389/fmed.2018.00263

\section{Potential of AKR1B10 as a Biomarker and Therapeutic Target in Type 2 Leprosy Reaction}

\author{
Cleverson T. Soares ${ }^{1 *}$, Luciana R. V. Fachin ${ }^{1}$, Ana P. F. Trombone ${ }^{2}$, Patricia S. Rosa ${ }^{3}$, \\ Cássio C. Ghidella ${ }^{4}$ and Andrea F. F. Belone ${ }^{1}$ \\ 1 Department of Anatomic Pathology, Instituto Lauro de Souza Lima, Bauru, Brazil, ${ }^{2}$ Department of Health Science, \\ Universidade do Sagrado Coração, Bauru, Brazil, ${ }^{3}$ Division of Research and Education, Instituto Lauro de Souza Lima, \\ Bauru, Brazil, ${ }^{4}$ Ambulatory of Leprosy, Jardim Guanabara Health Center, Rondonópolis, Brazil
}

The AKR1B10 (aldo-keto reductase family 1 member $\mathrm{B} 10$ ) gene has important functions in carcinogen-induced neoplasia. AKR1B10 is also expressed in type 2 reaction leprosy patients (R2). We measured the expression of AKR1B10 in the skin lesions of patients with leprosy by immunohistochemistry from biopsies that encompassed the spectrum of types of leprosy, based on the Ridley and Jopling classification [10 samples each of tuberculoid (TT), borderline tuberculoid (BT), mid-borderline (BB), and borderline lepromatous (BL) lesions; four samples of lepromatous lesions (LL)], reactional leprosy [14 samples of type 1 Reaction (R1) and 10 samples of type 2 Reaction (R2)], and biopsies from 9 healthy control $(\mathrm{HC})$ subjects. In addition, 46 lepromatous lesions (BL and $L \mathrm{~L}$ ), 45 lepromatous lesions in regression, and 115 R2 lesions were included. Eight of 10 R2 samples (80\%), 3 of 46 active $B L$ and LL samples (6\%), 23 of $45 \mathrm{BL}$ and $\mathrm{LL}$ samples in regression (51\%), and 107 of 115 R2 samples (93\%) were positive for AKR1B10, differing significantly between all groups $(p<0.05)$. AKR1B10 expression was highest in the cytoplasm of macrophages. Thus, AKR1B10 is overexpressed on the lepromatous side (BL and $\mathrm{LL}$ ) in samples that are in regression, especially type 2 reaction-associated lesions, rendering it a potential marker of type 2 reactional episodes of leprosy and a target of drugs against reactional episodes.

Keywords: leprosy, reaction type 2, AKR1B10, biomarker, therapeutic target

\section{INTRODUCTION}

The AKR1B10 (aldo-keto reductase family 1 member B10) gene encodes a member of the aldoketo reductase superfamily, which consists of over 40 enzymes and proteins $(1,2)$. Its ability to reduce several aldehydes and its expression in the luminal cells of the gastrointestinal mucosa suggest that it mediates the detoxification of reactive aldehydes in digested food before nutrients are distributed to other organs (1). AKR1B10 interferes with cell survival by modulating lipid synthesis, mitochondrial function, and oxidative status, implying that it is an important protein in cell survival mechanisms (3). In the intestine, AKR1B10 is expressed specifically by the epithelial cells and is secreted into the lumen (4). It is also commonly expressed in the adrenal gland and might have a significant function in liver carcinogenesis. AKR1B10 is overexpressed in neoplasms of various organs and systems, such as the lung, breast, gastrointestinal tract, pancreas, head, and neck $(4,5)$. 
Overexpression of AKR1B10 is an initial event in the carcinogenesis of lung, liver, pancreatic, and breast cancer (6-10). Based on its overexpression in neoplastic cells, it is considered a tumor marker, and it can be detected in situ and in the blood (11-13). Alterations in AKR1B10 expression have been observed in non-neoplastic diseases, such as atopic dermatitis and diabetes $(14,15)$. In vitro studies have shown that AKR1B10 is present in the lysosomes and is secreted with cathepsin $\mathrm{D}$, a lysosomal marker $(16,17)$

Leprosy is a chronic infectious disease that is caused by Mycobacterium leprae (M. leprae) (18). It is an important public health issue in Asian, African, and South American countries (18). Because leprosy is a spectral disease that can evolve over many years or decades, its clinicopathological characteristics change slowly but continuously-sometimes with overlapping clinical and histopathological patterns $(19,20)$.

According to the Ridley and Joplin (R\&J) classification system, leprosy is subdivided into polar [tuberculoid (TT) and lepromatous (LL)] and intermediate forms [borderline tuberculoid (BT), mid-borderline (BB), and borderline lepromatous $(\mathrm{BL})] ;(21)$. It is believed that this behavior is caused by the low antigenicity of $M$. leprae, which stimulates low-intensity immunocellular reactions. However, episodes of abrupt onset of an inflammatory reaction in cutaneous neural lesions, which are generally more intense and potentially destructive, can be intercalated into the evolution of the disease.

These episodes are called leprosy reactions (19, 20, 22), of which there are 2 types. The type 1 reaction (R1) occurs in patients with varying degrees of preservation of cellular immunity, specific to $M$. leprae. The type 2 reaction (R2), corresponding to erythema nodosum leprosum and its variants develops in patients in whom little cellular immunity is preserved or absent (19, 20, 22). During these episodes, neurological lesions usually worsen with the destruction of the neural branches and tissues, which can cause functional disabilities and permanent sequelae $(19,20,22)$.

There are few studies on the association between AKR1B10 and leprosy. Recently, we reported that AKR1B10 was overexpressed in skin biopsies of patients with leprosy, especially those with the type 2 reaction (23). Thus, AKR1B10 has emerged as a potential marker of and therapeutic target in type 2 reactions. The aim of this study was to examine the expression of AKR1B10 in leprosy skin lesions by immunohistochemistry (IHC) and identify the cells in granulomas and skin tissue that express this marker.

\section{MATERIALS AND METHODS}

The experimental design is shown in Figure 1. IHC was performed as described using the EnVision indirect method kit (Dako, California, USA) following the manufacturer's recommendations. Monoclonal anti-AKR1B10 was used (EPR14421 clone, 1:500, Abcam, Cambridge, UK) to measure the expression of AKR1B10 in all samples. Initially, as an external positive control, the antibody was tested in samples of the amygdala, duodenal mucosa, well-differentiated hepatocellular carcinoma, and pancreatic ductal adenocarcinoma (Figures 1A-D).

After verifying the activity of anti-AKR1B10 in these controls, we evaluated the expression of AKR1B10 in the same samples of leprosy lesions and healthy skin as in our previous study. We had observed upregulation of AKR1B10 mRNA in R2 samples compared with biopsies of other leprosy types along the R\&J spectrum [HC (9 samples); TT, BT, BB, and BL (10 samples each); LL (4 samples)], R1 biopsies (14 samples), and R2 biopsies (10 samples) [(23); Figures 1E-T]. In a subsequent analysis, we verified our observations in skin biopsies from patients with lesions on the lepromatous side of the R\&J spectrum (BL and LL) before treatment (L side; $n=46$ ) and after initiation of treatment (L side-T) $(n=45)$ and those with type 2 reactional lesions $(n=$ 115; Figures 1U-W).

All samples were obtained from the Lauro de Souza Lima Institute pathology laboratory archives of leprosy cases that were diagnosed from 2005 to 2016 . The L side samples were obtained from patients with borderline lepromatous (BL) or lepromatous (LL) lesions, as confirmed by Fite-Faraco staining and showing well-stained solid bacilli before undergoing treatment. The $\mathrm{L}$ side $\mathrm{T}$ samples belonged to patients with lepromatous lesions (BL and $\mathrm{LL}$ ) in the initial phase of their multidrug therapy or up to 2 years after the end of treatment. The R2 samples were also taken from patients with lesions on the lepromatous side (BL and LL) of the R\&J spectrum after they had begun treatment but with the characteristic histological features that are associated with the type 2 reaction (Figure 2). Sequential sections of all samples were subjected to hematoxylin-eosin (HE) and Fite-Faraco staining (for bacilloscopy) and then IHC using anti-AKR1B10. All cases were reviewed to confirm their bacilloscopic index, their R\&J classification, the presence of $\mathrm{R} 2$, and regression status $(19,21,22)$. Samples from patients who had undergone 5 or more years of treatment were not included.

This study adhered to the Declaration of Helsinki and was approved by the Research Ethics Committees of Lauro de Souza Lima Institute, certified by CAAE 79664917.9.0000.5475. The clinical data of the patients are detailed in Supplement $\mathbf{1 .}$

When the skin samples or the inflammatory cells in granulomas showed a weak/unclear signal (rated $1+$ on a scale of 0 to $3+$ ) or a lack of staining for AKR1B10, they were considered negative (Figures 3A,B). Moderate or strong $(2+/ 3+)$ staining for AKR1B10 was considered positive in these samples (Figures 3C,D). The sample slides were first scanned at low magnification $(10 \times)$ and then at high magnification $(40 \times)$ to identify the immunostained cells (Figures 3A-D). The data on the differential expression of AKR1B10 in each sample were evaluated by two observers. The expression was examined in the following tissues: epidermis, cutaneous adnexal glands, interstitium, blood vessel walls, endothelium, subcutaneous adipose tissue, and cells in the inflammatory infiltrates that constituted the leprosy granulomas. Comparisons between groups were made using Fisher's exact test (GraphPad Prism 6.05; GraphPad Software, Inc., San Diego, California, USA; Figures 3E,F). 


\section{Expression of AKR1B10 in control samples}
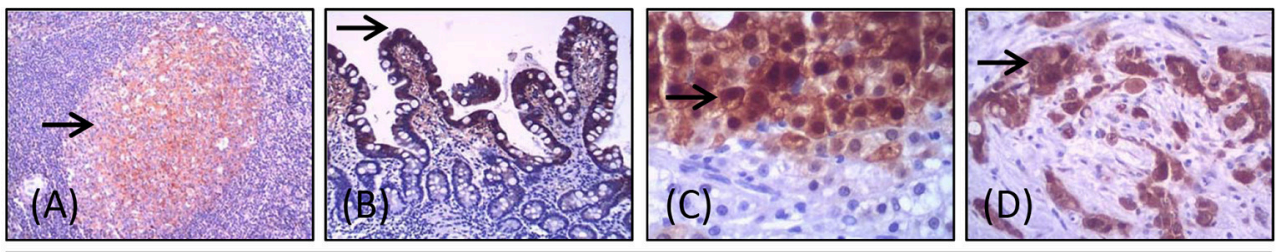

$\downarrow$

Expression of AKR1B10 in samples of leprosy forms and reactions
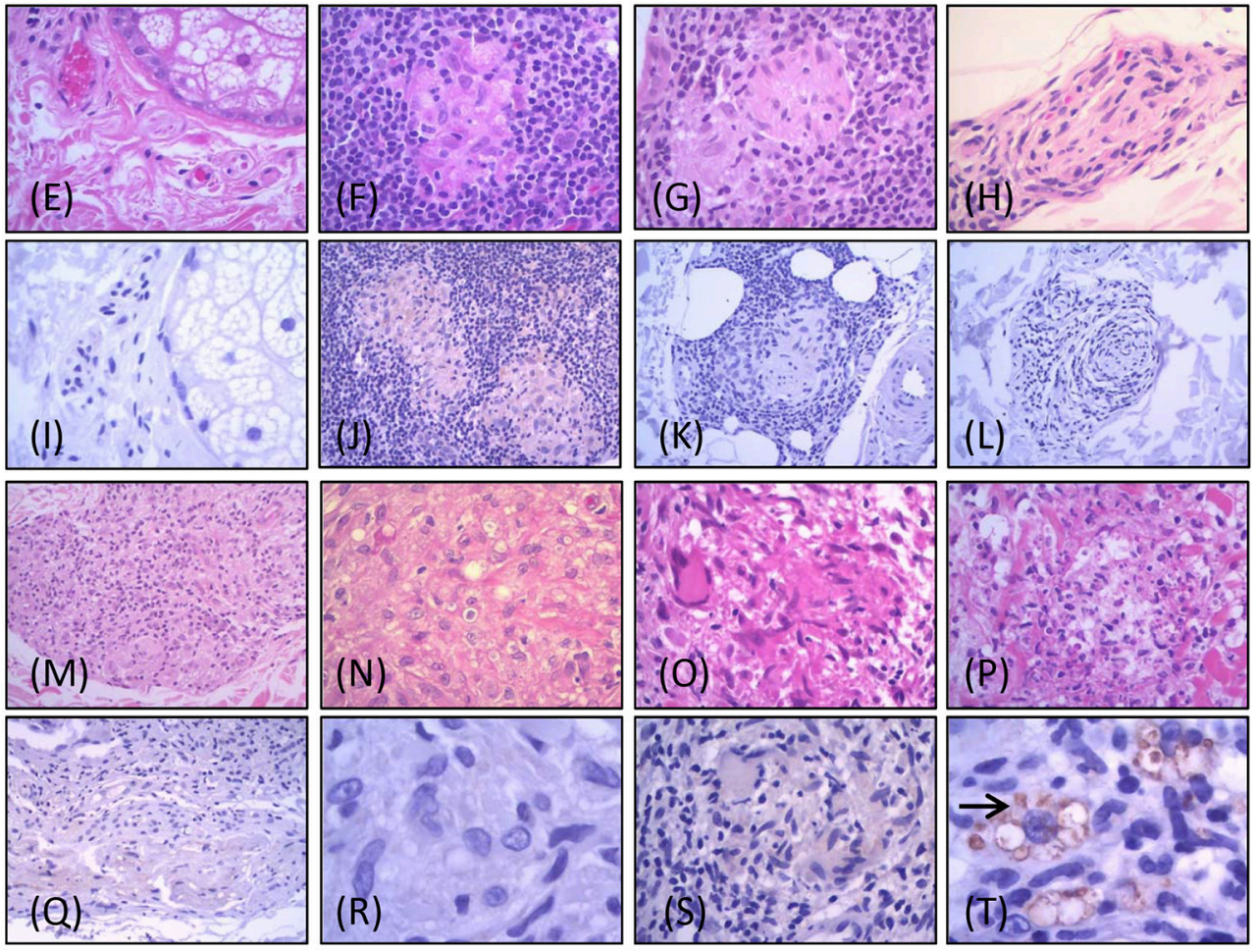

$\psi$

$(\mathrm{BL}+\mathrm{LL})$ in activity

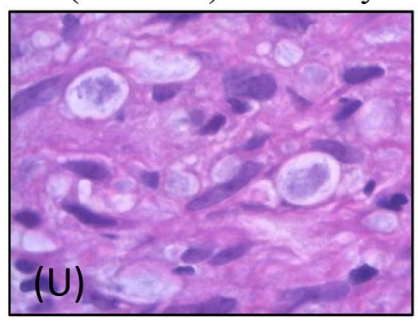

$(\mathrm{BL}+\mathrm{LL})$ in regression
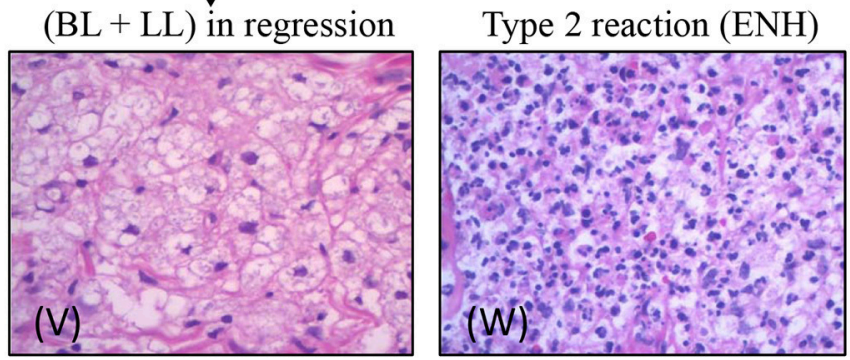

FIGURE 1 | Experimental design of the project and expression of AKR1B10 (marked by $\rightarrow$ ). Positive expression of AKR1B10 in controls: cells of the follicular center of the amygdala (A), columnar cells in the duodenal mucosa (B), in well-differentiated cells of hepatocellular carcinoma (C), and in the carcinoma of pancreatic ducts (D) Negative expression (absence or weak expression) in samples of healthy controls (HC) (E,I) and in different forms of leprosy [TT (F,J); BT (G,K); BB (H,L); BL (M,Q);

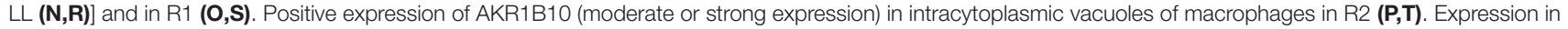
samples of lepromatous side (BL and $L L)$ lesions in activity ( $L$-side) $(n=46$; $\mathbf{U})$, lepromatous side lesions $(B L$ and $L L)$ in regression $(L$ side $T)(n=45$; $\mathbf{V})$, and $R 2$ lesions $(n=115 ; \mathbf{W})$.

\section{RESULTS}

The data from this study are summarized in Figures 1-4. The control samples showed moderate $(2+)$ to strong $(3+)$ immunostaining of AKR1B10 (Figures 1A-D). Of the leprosy lesions and healthy skin samples from our previous study, which reported transcriptional upregulation of $A K R 1 B 10$ in $\mathrm{R} 2$ samples, all healthy controls $(n=9)$, leprosy lesion samples from the 

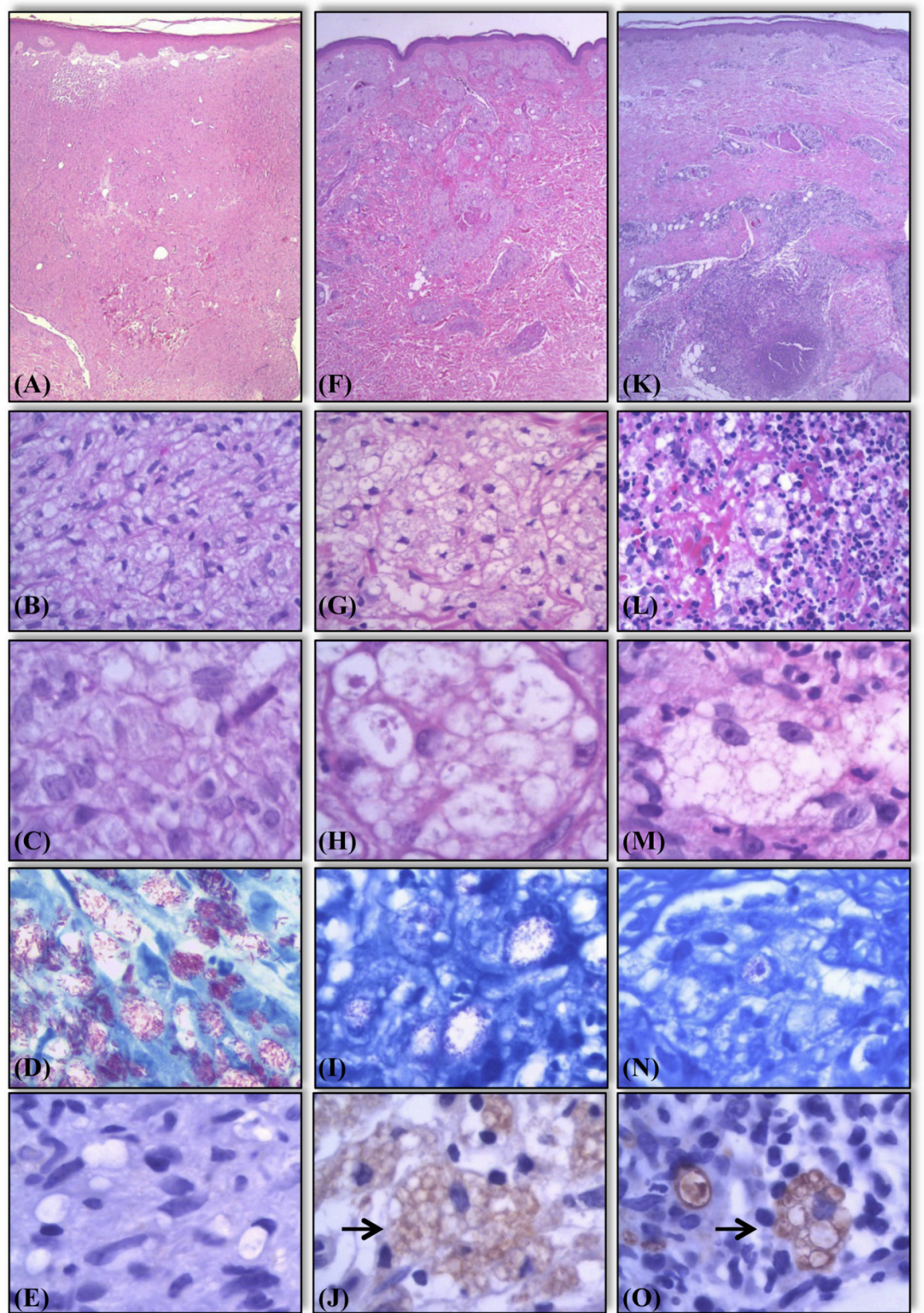

FIGURE 2 | Histological and bacilloscopic characteristics of lesions of lepromatous side in activity, lepromatous side in regression, and R2 lesions. Active lesions with vacuolated macrophages containing a large number of solid bacilli (A-D). Lesions in regression showing multivacuoled macrophages containing a large number of multifragmented bacilli (F-I). R2 lesions, the macrophages display characteristics similar to those seen in lesions in regression undergoing an influx of neutrophils $(\mathbf{K}-\mathbf{N})$. Expression of AKR1B10 in the same samples. Negative expression in samples of lepromatous side in activity (E). Positive expression (marked by $\rightarrow$ ) in

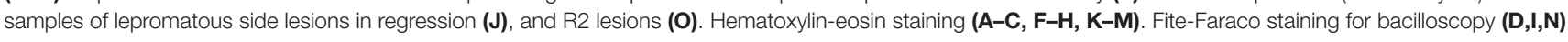

R\&J spectrum (10 TT, BT, BB, and BL samples; 4 LL samples), and R1 samples $[n=14$; Figures 1E-S; (23)] were negative. However, AKR1B10 immunostaining was positive in 8 of 10 (8/10) R2 samples, corresponding to $80 \%$ positivity (Figure 1T). By pairwise comparison between several groups, there was a significant difference in AKR1B10 positivity (R2 vs. HC, R2 vs. TT, R2 vs. BT, R2 vs. BB, and R2 vs. BL, $p=0.0007$; 2 vs. LL, $p=0.015$; and R1 vs. R2, $p<0.0001$; Figures 3E,F).

Among L side lesions, 3 of 46 (3/46) samples were positive by immunostaining, corresponding to $6 \%$ of all samples. L side 

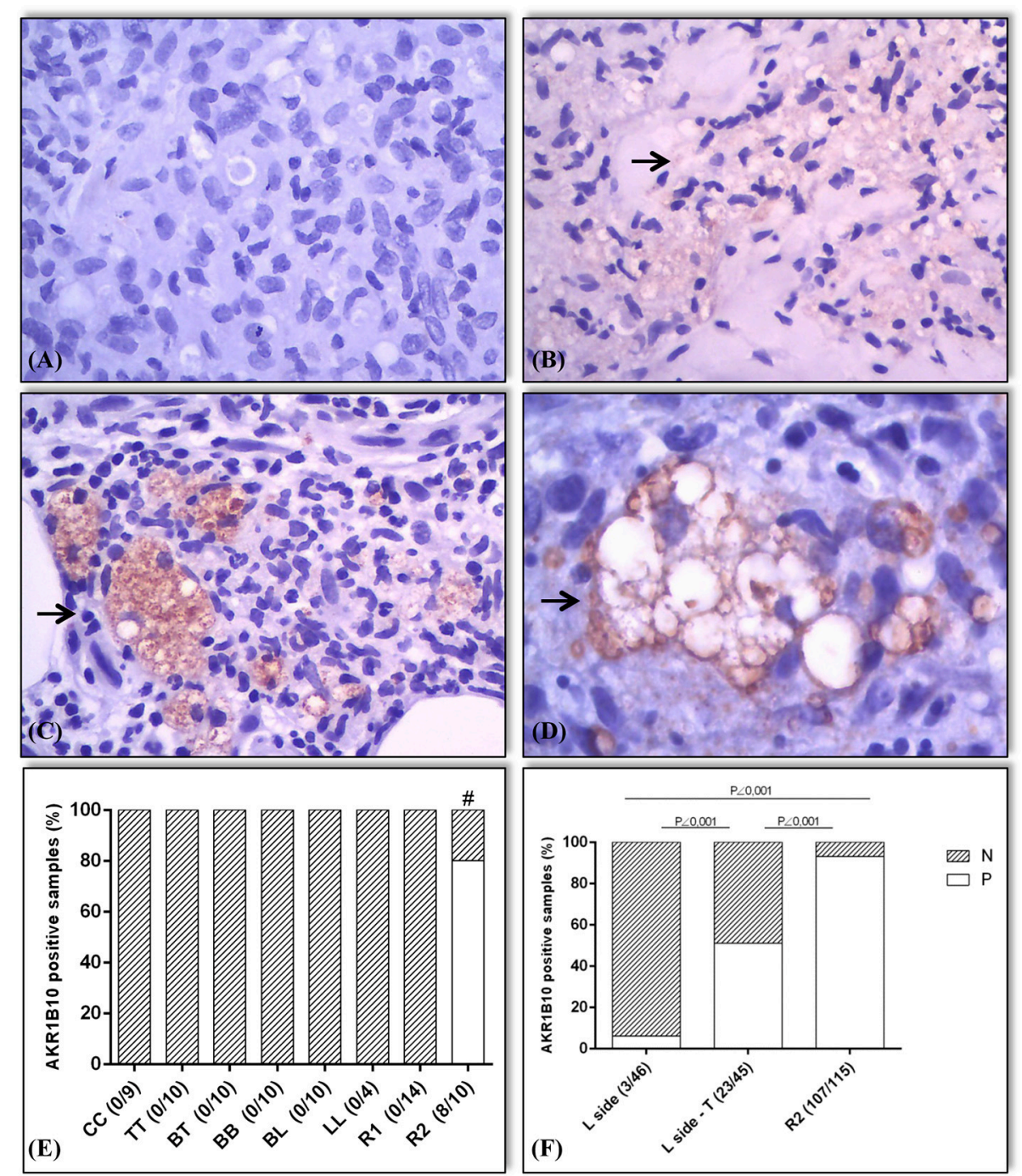

FIGURE 3 | Expression pattern (marked by $\rightarrow$ ) and expression values of AKR1B10 in positive samples. The samples were classified as negative for AKR1B10 when its expression was absent (0) or weak (1+; $\mathbf{A}, \mathbf{B})$. Positive expression of AKR1B10 $(2+$ or $3+$ on the scale; $\mathbf{C , D})$. In the preliminary assessment (E), immunostaining was negative in all samples of $\mathrm{HC}, \mathrm{TT}, \mathrm{BT}, \mathrm{BB}, \mathrm{BL}, \mathrm{LL}$, and R1. It was positive in 8 out of 10 samples of R2 (80\%), with a significant difference between groups (\# R2 vs. $\mathrm{HC}$, R2 vs. $T$, R2 vs. $\mathrm{BT}$, R2 vs. $\mathrm{BB}$, and $\mathrm{R} 2$ vs. $\mathrm{BL}$, all with $p=0.0007$; R2 vs. $\mathrm{LL}, p=0.015 ; \mathrm{R} 1 \mathrm{vs}$. $\mathrm{R} 2, p<0.0001$ ). In later evaluation (F), there was positive expression in 3 out of 46 active lepromatous ( $B L$ and $L L)$ samples (6\%) ( $L$ side), 23 out of 45 samples of $B L+L L$ in regression after treatment $(L$ side- $T)(51 \%)$, and 107 out of 115 samples of R2 (93\%), with a significant difference between the $L$ side samples and $L$ side- $T(p<0.0001)$, R2 and $L$ side $(p<0.0001)$, and between R2 and $L$ side-T groups $(p<0.0001)$.

$\mathrm{T}$ lesions were positive in 23 of 45 (23/45) samples (51\%). Similarly, among R2 lesions, 107 of $115(+107 / 115)$ samples showed positivity (93\%) (Figures 2, 3F). Moreover, there was a significant difference in positivity between $\mathrm{L}$ side and $\mathrm{L}$ side $\mathrm{T}$ groups ( $p<0.0001), \mathrm{R} 2$ and L side groups $(p<0.0001)$, and $\mathrm{R} 2$ and L side T groups ( $p<0.0001$; Figure $3 F)$.

Next, we examined the expression of AKR1B10 in various tissues and inflammatory infiltrates of leprosy but failed to detect it in any component of the skin or subcutaneous tissue. AKR1B10 was observed nearly exclusively in the cytoplasm of macrophages. Some macrophages showed intense expression of AKR1B10 in their intracytoplasmic vesicles, such as lysosomes and phagosomes (Figures 1S-T, 2J-O, 3C,D, 4F). In several samples, AKR1B10 was expressed in the neural branches that were involved in leprosy-associated inflammatory processes. In these cases, morphologically, AKR1B10 was predominantly expressed in the macrophages that permeated the neural branches that had been infected by parasites. Supplement 2 shows the data on the expression of AKR1B10 in these samples and their respective statistical values.

AKR1B10 expression was not homogeneous between clusters of inflammatory cells (Figure 4). In R2 granulomas, AKR1B10 was detected mostly in multivacuoled macrophages involving 


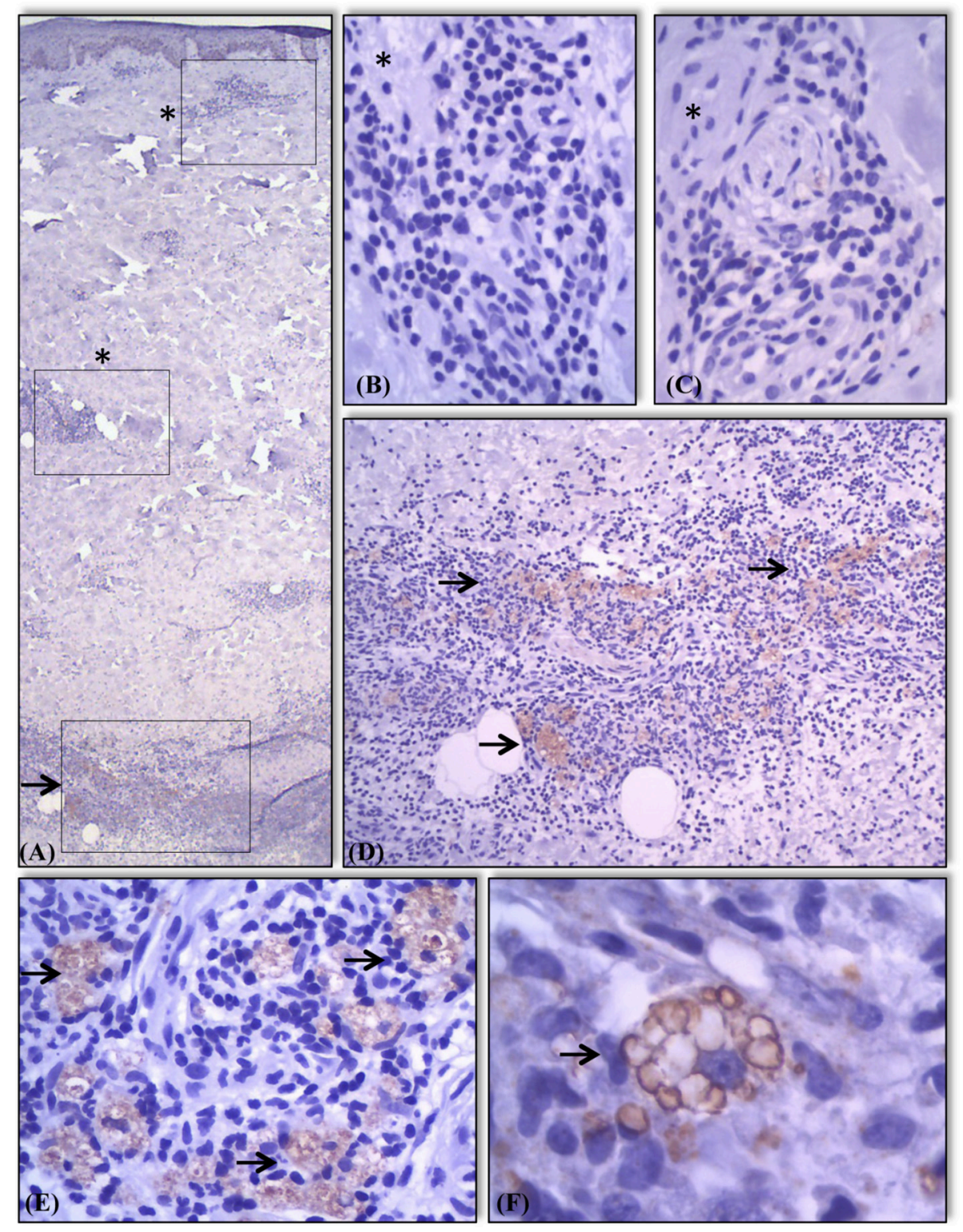

FIGURE 4 | Expression of AKR1B10 in different components of skin and granulomas in the samples. Absence (marked with a*) of AKR1B10 expression in most components of skin and in some granulomas (A-C). Positive expression (marked with a $\rightarrow$ ) in granulomas present in other areas of the same sample (A,D). Moderate or strong expression of marker almost exclusively in the cytoplasm of macrophages present in lesions of lepromatous lesions in regression (E) and R2 (F) showing staining of intracytoplasmatic vacuoles/lysosomes (F).

a central area of microabcesses and some were detected within microabscesses (Figures 4D,E). AKR1B10 was absent from lymphocytes, plasmocytes, neutrophils, and other cells that constituted the leprosy granulomas (Figure 4).

\section{DISCUSSION}

AKR1B10 regulates several metabolic processes, based on its function in the metabolism of lipids and aldehydes $(1,2)$. Its dysregulation is associated with the development of various cancers. The function of $A K R 1 B 10$ in the development of inflammatory and infectious diseases is unknown $(4,9,14,15)$.

The mechanism of AKR1B10 varies between neoplasias (11). In pancreatic ductal neoplasms, AKR1B10 is overexpressed in precursor lesions and invasive carcinoma. Further, silencing of AKR1B10 is associated with greater apoptosis and decreased activation of KRAS and many of its effectors (7). In hepatocellular carcinoma, the expression of AKR1B10 is regulated through the activation of $\beta$-catenin signaling (24). In breast carcinoma, AKR1B10 promotes the migration and invasion of neoplastic cells by stimulating ERK signaling $(25,26)$. Inhibition of 
AKR1B10 constrains the growth of pancreatic cancer through modulation of the KRAS-E-cadherin pathway (27). Moreover, AKR1B10 participates in the activation of several mechanisms of drug resistance during the treatment of various cancers $(5,7,28,29)$.

There are few reports on the participation of AKR1B10 in the development of non-neoplastic diseases. The significantly higher expression of AKR1B10 in peripheral blood mononuclear cells (PBMCs) in diabetic nephropathy patients vs. healthy controls suggests that AKR1B10 mediates the development and progression of diabetic nephropathy (14). With regard to infectious diseases, AKR1B10 is overexpressed in hepatitis B and $\mathrm{C}$ and is linked to the development of hepatocellular carcinoma $(9,30-32)$. In inflammatory bowel disease, the loss of AKR1B10 correlates with chronic colitis and the development of colitisrelated carcinoma (4).

There is little information on the function of AKR1B10 in leprosy. We recently published a study on the expression of genes in skin biopsy samples along the R\&J spectrum of the disease and in its reactional forms (23). AKR1B10 was upregulated only in R2 samples. Also, several miRNAs were differentially expressed in the spectral and reactional forms of leprosy. Based on our analysis of mi/mRNA expression, we noted that the expression patterns of hsa-miRNA-142-3p and AKR1B10 opposed each other (33). These results indicate that the expression of AKR1B10 is regulated by microRNA (33). The regulation of mRNA expression by miRNAs is complex, and there are also other mechanisms of epigenetic regulation of gene expression, necessitating further studies to determine whether there is an interaction between hsa-miR-142-3p and AKR1B10 in leprosy.

In macrophages, AKR1B10 expression was nearly confined to the cytoplasm, especially around the membranes of intracytoplasmic vacuoles (lysosomes; Figures 1-4). In normal tissues and neoplasms, AKR1B10 is expressed in various types of cells-primarily epithelial lineage cells-whereas in leprosy, it is expressed in macrophages. In macrophages, AKR1B10 is secreted through lysosomes through a non-classical pathway, increasing its level in the serum of patients with breast cancer (16). AKR1B10 localizes to the cytoplasm and translocates to lysosomes via heat shock protein 90 (HSP90), a chaperone molecule. After fusion of the lysosome to the plasma membrane, AKR1B10 is released into the interstitium and then into the bloodstream and thus can be measured in serum (17)

There is a little knowledge about the pathophysiological mechanisms that trigger an R2 reaction $(19,21)$. R2 occurs in lepromatous lesions (BL and LL), usually after treatment is initiated. It is believed that during bacillary fragmentation, which can be spontaneous or caused by multidrug therapy, antigens that stimulate the production of antibodies are released into the blood and interstitial fluids $(19,21)$. Due to unknown mechanisms of the abrupt release of antigens from macrophages, an antigen-antibody reaction occurs in the interstitium, accompanied by complement fixation and stimulation, developing into an acute inflammatory reaction with varying intensities $(19,21)$.
The histological features of $\mathrm{R} 2$ reflect an acute or subacute inflammatory reaction in the foci of lepromatous granulomas during regression (19). In general, R2 is a well-defined example of an acute inflammatory reaction, comprising vascular dilation, endothelial swelling, and serofibrinous and neutrophilic exudation, disrupting pre-existing granulomas. The more intense processes include the development of thrombi in the venocapillary territory, vasculitis, and the formation of microabscesses (19). Like most antigens in the extravascular space, the antigen-antibody reaction and complement fixation occur in parasitized tissues, triggering an acute inflammatory reaction in which neutrophils undergo chemotaxis and subsequently migrate to the interstitium to induce phagocytosis and destruction of the immune complexes (19).

The function of AKR1B10 in leprosy is unknown. It is unclear whether its expression in macrophages is associated with the development of R2. Nonetheless, our results indicate that AKR1B10 predominates in macrophages on the start of treatment (L-side $\mathrm{T}$ ) and in R2 lesions. It is possible that the expression of AKR1B10 in the lysosomes of macrophages is phenomenon precipitating event, resulting from the intense fragmentation of bacilli by chemotherapy, and triggers an R2 reaction. Our data show that the macrophages in leprosy lesions that are in regression after treatment (showing no evidence of R2) express AKR1B10, which is nearly absent in active macrophages in the lesion (Figure 3).

There are significant histological differences between active lesions and lesions that are in regression. In active lepromatous BL and LL lesions, macrophages have an M2 immunoprofile, with an eosinophilic, vacuolated cytoplasm that contains many solid, well-stained bacilli and fewer fragmented bacilli in the vacuoles and cytoplasm (Figures 2A-E). At the outset of treatment, there is intense and progressive multivacuolation of the cytoplasm in macrophages. These vacuoles have distinct morphological features and a lysosomal profile by IHC, containing many multifragmented bacilli with varying intensities of Fite-Faraco staining. It is unknown how bacilli interfere with the expression of AKR1B10 in parasitized macrophages. However, our findings indicate that the predominance of bacilli in active lesions blocks AKR1B10 expression in the cytoplasm and the formation of vacuoles in macrophages in these active lesions. When treatment begins, the fragmentation of bacilli disrupts this balance; consequently, the macrophages express AKR1B10.

The expression of AKR1B10 could lead to the exposure of antigens on the surface of macrophages or enhance the fusion of intracytoplasmic vacuoles that contain a large number of fragmented bacilli in the plasma membrane and then release them into the interstitium, or it might promote the disintegration of macrophages. If AKR1B10 functions in the development of R2, substances that inhibit it could act on parasitized macrophages that retain fragmented bacilli in the lysosomes, thus retarding bacterial clearance and blocking the factors that trigger the onset of reactional episodes or decreasing the frequency and intensity of R2. 
Thus, even if R2 develops, the reaction episodes would be less intense, allowing better management of reactions and mitigation of clinical sequelae, which are caused primarily by intense reaction episodes that destroy neural branches and tissues.

Leprosy reactions are significant in the development of leprosy $(19,20)$. There is no marker, serological or in situ, that identifies or predicts the occurrence of reactional episodes (20). The most effective drug that is used to treat R2 is thalidomide (34). Because it is teratogenic, its use is restricted, and it is sometimes replaced with less effective drugs, such as corticosteroids, which have major side effects [immunosuppression, diabetes, obesity, osteoporosis, glaucoma, etc.; (35)].

Because AKR1B10 has been identified in the blood as a cancer marker, it is possible that it has a similar function in R2 (13). Whether anti-AKR1B10 drugs block the activation of R2 or eventually reduce the intensity and frequency of reactional episodes should be examined. There are several reports on various types of drugs, from herbal drugs to monoclonal antibodies, with anti-AKR1B10 activity (27, 30, 32, 36-50).

In summary, $A K R 1 B 10$ is expressed in the skin lesions of patients with leprosy, predominantly in lepromatous lesions that are in regression, especially those that are associated with R2. Further studies should determine whether AKR1B10 is important for triggering the $\mathrm{R} 2$ reaction and whether it can be used as a marker or therapeutic target for the prevention and clinical management of R2 episodes of leprosy.

\section{REFERENCES}

1. Cao D, Fan ST, Chung SS. Identification and characterization of a novel human aldose reductase-like gene. J Biol Chem. (1998) 273:11429-35. doi: 10.1074/jbc.273.19.11429

2. Penning TM. The aldo-keto reductases (AKRs): overview. Chem Biol Interact. (2015) 234:236-46. doi: 10.1016/j.cbi.2014.09.024

3. Wang C, Yan R, Luo D, Watabe K, Liao DF, Cao D. Aldo-keto reductase family 1 member B10 promotes cell survival by regulating lipid synthesis and eliminating carbonyls. J Biol Chem. (2009) 284:26742-8. doi: 10.1074/jbc.M109.022897

4. Shen Y, Liao DF, Cao D. AKR1B10 in gastrointestinal diseases. Aging (2015) 7:221-2. doi: 10.18632/aging.100737

5. Huang L, He R, Luo W, Zhu YS, Tan T, Zhang X, et al. Aldoketo reductase family 1 member B10 inhibitors: potential drugs for cancer treatment. Recent Pat Anticancer Drug Discov. (2016) 11:184-96. doi: $10.2174 / 1574892811888160304113346$

6. Wang R, Wang G, Ricard MJ, Ferris B, Strulovici-Barel Y, Salit J, et al. Smoking-induced upregulation of AKR1B10 expression in the airway epithelium of healthy individuals. Chest (2010) 138:1402-10. doi: 10.1378/chest.09-2634

7. Chung YT, Matkowskyj KA, Li H, Bai H, Zhang W, Tsao MS, et al. Overexpression and oncogenic function of aldo-keto reductase family 1 B10 (AKR1B10) in pancreatic carcinoma. Mod Pathol. (2012) 25:758-66. doi: 10.1038/modpathol.2011.191

8. Ma J, Luo DX, Huang C, Shen Y, Bu Y, Markwell S, et al. AKR1B10 overexpression in breast cancer: association with tumor size, lymph node metastasis and patient survival and its potential as a novel serum marker. Int J Cancer. (2012) 131:E862-71. doi: 10.1002/ijc.27618

9. Sato S, Genda T, Hirano K, Tsuzura H, Narita Y, Kanemitsu Y, et al. Upregulated aldo-keto reductase family 1 member B10 in chronic hepatitis

\section{AUTHOR CONTRIBUTIONS}

$\mathrm{CS}$ and $\mathrm{AB}$ conceived the project. $\mathrm{CG}$ and $\mathrm{CS}$ performed clinical evaluation of patients and performed biopsy procedures. CS performed histopathological and immunohistochemical analyses. CS, AB, AT, LF, and PR were responsible for data analysis. CS and PR wrote the manuscript. All authors reviewed the paper and provided their inputs.

\section{FUNDING}

This work was funded by the São Paulo State Research Foundation (FAPESP) Process no. 2010/19286-3.

\section{ACKNOWLEDGMENTS}

We are grateful to Daniel D. Amorim, Everson Moretti, Nelci A. Vieira, Osmar de A. Francisco, Ana Lúcia de Oliveira, Fabiana Aparecida de Camargo Bertonha, and Neusa B. Coelho for technical assistance. We also give special thanks to the dermatologists Somei Ura and Jaison Antonio Barreto.

\section{SUPPLEMENTARY MATERIAL}

The Supplementary Material for this article can be found online at: https://www.frontiersin.org/articles/10.3389/fmed. 2018.00263/full\#supplementary-material

C: association with serum alpha-fetoprotein and hepatocellular carcinoma. Liver Int. (2012) 32:1382-90. doi: 10.1111/j.1478-3231.2012.02827.x

10. Tsuzura H, Genda T, Sato S, Murata A, Kanemitsu Y, Narita Y, et al. Expression of aldo-keto reductase family 1 member b10 in the early stages of human hepatocarcinogenesis. Int J Mol Sci. (2014) 15:6556-68. doi: 10.3390/ijms15046556

11. Kapoor S. AKR1B10 and its emerging role in tumor carcinogenesis and as a cancer biomarker. Int J Cancer (2013) 132:495-7. doi: 10.1002/ijc.27685

12. Matkowskyj KA, Bai H, Liao J, Zhang W, Li H, Rao S, et al. Aldoketoreductase family $1 \mathrm{~B} 10$ (AKR1B10) as a biomarker to distinguish hepatocellular carcinoma from benign liver lesions. Hum Pathol. (2014) 45:834-43. doi: 10.1016/j.humpath.2013.12.002

13. Wang Y, Li Y, Wang T, Gu J, Zhao J, Pan Z. Detection of AKR1B10 in peripheral blood by anti-AKR1B10-conjugated CdTe/CdS quantum dots. Clin Lab. (2015) 61:1267-74. doi: 10.7754/Clin.Lab.2015.150203

14. Shaw N, Yang B, Millward A, Demaine A, Hodgkinson A. AKR1B10 is induced by hyperglycaemia and lipopolysaccharide in patients with diabetic nephropathy. Cell Stress Chaperones (2014) 19:281-7. doi: 10.1007/s12192-013-0455-6

15. Ghosh D, Ding L, Sivaprasad U, Geh E, Biagini Myers J, Bernstein JA, et al. Multiple transcriptome data analysis reveals biologically relevant atopic dermatitis signature genes and pathways. PLoS ONE (2015) 10:e0144316. doi: 10.1371/journal.pone.0144316

16. Luo DX, Huang MC, Ma J, Gao Z, Liao DF, Cao D. Aldo-keto reductase family 1 , member B10 is secreted through a lysosome-mediated non-classical pathway. Biochem J. (2011) 438:71-80. doi: 10.1042/BJ20 110111

17. Luo D, Bu Y, Ma J, Rajput S, He Y, Cai G, et al. Heat shock protein 90- $\alpha$ mediates aldo-keto reductase 1B10 (AKR1B10) protein secretion through secretory lysosomes. J Biol Chem. (2013) 288:36733-40. doi: 10.1074/jbc.M113.514877 
18. World Health Organization. Global leprosy update, 2015: time for action, accountability and inclusion. Wkly Epidemiol Rec. (2015) 91:405-20.

19. Fleury, R. N. Patologia e manifestações viscerais. In: Opromolla DVA, editor. Noções de Hansenologia. 2nd ed. Bauru: Centro de Estudo Dr. Reynaldo Quagliato, Instituto Lauro de Souza Lima. (2000). p. 63-71.

20. Opromolla DVA. Noções de Hansenologia. Bauru: Centro de Estudos Reynaldo Quagliato (2000).

21. Ridley DS, Jopling WH. Classification of leprosy according to immunity. A five-group system. Int J Lepr Other Mycobact Dis. (1966) 34:255-73.

22. Fachin LR, Soares CT, Belone AF, Trombone AP, Rosa PS, Guidella CC, et al. Immunohistochemical assessment of cell populations in leprosyspectrum lesions and reactional forms. Histol Histopathol. (2017) 32:385-96. doi: 10.14670/HH-11-804

23. Belone AF, Rosa PS, Trombone AP, Fachin LR, Guidella CC, Ura S, et al. Genome-Wide Screening of mRNA Expression in Leprosy Patients. Front Genet. (2015) 6:334. doi: 10.3389/fgene.2015.00334

24. Liu TA, Jan YJ, Ko BS, Wu YJ, Lu YJ, Liang SM, et al. Regulation of aldo-keto-reductase family 1 B10 by $14-3-3 \varepsilon$ and their prognostic impact of hepatocellular carcinoma. Oncotarget (2015) 6:38967-82. doi: 10.18632/oncotarget.5734

25. Li J, Guo Y, Duan L, Hu X, Zhang X, Hu J, et al. AKR1B10 promotes breast cancer cell migration and invasion via activation of ERK signaling. Oncotarget (2017) 8:33694-703. doi: 10.18632/oncotarget.16624

26. Reddy KA, Kumar PU, Srinivasulu M, Triveni B, Sharada K, Ismail A, et al. Overexpression and enhanced specific activity of aldoketo reductases (AKR1B1 \& AKR1B10) in human breast cancers. Breast (2017) 31:137-43. doi: 10.1016/j.breast.2016.11.003

27. Zhang W, Li H, Yang Y, Liao J, Yang GY. Knockdown or inhibition of aldo-keto reductase $1 \mathrm{~B} 10$ inhibits pancreatic carcinoma growth via modulating Kras-E-cadherin pathway. Cancer Lett. (2014) 355:273-80. doi: 10.1016/j.canlet.2014.09.031

28. Matsunaga T, Suzuki A, Kezuka C, Okumura N, Iguchi K, Inoue I, et al. Aldo-keto reductase $1 \mathrm{~B} 10$ promotes development of cisplatin resistance in gastrointestinal cancer cells through down-regulating peroxisome proliferator-activated receptor- $\gamma$-dependent mechanism. Chem Biol Interact. (2016) 256:142-53. doi: 10.1016/j.cbi.2016.07.008

29. Chewchuk S, Guo B, Parissenti AM. Alterations in estrogen signalling pathways upon acquisition of anthracycline resistance in breast tumor cells. PLoS ONE (2017) 12:e0172244. doi: 10.1371/journal.pone.0172244

30. Takemura M, Endo S, Matsunaga T, Soda M, Zhao HT, El-Kabbani O, et al. Selective inhibition of the tumor marker aldo-keto reductase family member 1 B10 by oleanolic acid. J Nat Prod. (2011) 74:1201-6. doi: 10.1021/np200118q

31. Sato S, Genda T, Ichida T, Murata A, Tsuzura H, Narita Y, et al. Impact of aldo-keto reductase family 1 member B10 on the risk of hepatitis $\mathrm{C}$ virusrelated hepatocellular carcinoma. Gastroenterol Hepatol. (2016) 31:1315-22. doi: 10.1111/jgh.13295

32. Murata A, Genda $\mathrm{T}$, Ichida $\mathrm{T}$, Amano $\mathrm{N}$, Sato S, Tsuzura $\mathrm{H}$, et al. Pretreatment AKR1B10 expression predicts the risk of hepatocellular carcinoma development after hepatitis C virus eradication. World J Gastroenterol. (2016) 22:7569-78. doi: 10.3748/wjg.v22.i33.7569

33. Soares CT, Trombone APF, Fachin LRV, Rosa PS, Ghidella CC, Ramalho RF, et al. Differential expression of microRNAs in leprosy skin lesions. Front Immunol. (2017) 8:1035. doi: 10.3389/fimmu.2017.01035

34. Zhou S, Wang F, Hsieh TC, Wu JM, Wu E. Thalidomide-a notorious sedative to a wonder anticancer drug. Curr Med Chem. (2013) 20:4102-8. doi: 10.2174/09298673113209990198

35. Van Veen NHJ, Nicholls PG, Smith WCS, Richardus JH. Corticosteroids for treating nerve damage in leprosy. Cochrane Database Syst Rev. (2016) 5:CD005491. doi: 10.1002/14651858.CD005491.pub3

36. Loeffler-Ragg J, Mueller D, Gamerith G, Auer T, Skvortsov S, Sarg B, et al. Proteomic identification of aldo-keto reductase AKR1B10 induction after treatment of colorectal cancer cells with the proteasome inhibitor bortezomib. Mol Cancer Ther. (2009) 8:1995-2006. doi: 10.1158/1535-7163.MCT-08-0987

37. Matsunaga T, Endo S, Soda M, Zhao HT, El-Kabbani O, Tajima K, et al. Potent and selective inhibition of the tumor marker AKR1B10 by bisdemethoxycurcumin: probing the active site of the enzyme with molecular modeling and site-directed mutagenesis. Biochem Biophys Res Commun. (2009) 389:128-32. doi: 10.1016/j.bbrc.2009.08.107
38. Ruf TF, Quintes S, Sternik P, Gottmann U. Atorvastatin reduces the expression of aldo-keto reductases in HUVEC and PTEC. A new approach to influence the polyol pathway. Clin Invest Med. (2009) 32:E219-28. doi: 10.25011/cim.v32i3.6111

39. Endo S, Matsunaga T, Soda M, Tajima K, Zhao HT, El-Kabbani O, et al. Selective inhibition of the tumor marker AKR1B10 by antiinflammatory N-phenylanthranilic acids and glycyrrhetic acid. Biol Pharm Bull. (2010) 33:886-90. doi: 10.1248/bpb.33.886

40. Díez-Dacal B, Gayarre J, Gharbi S, Timms JF, Coderch C, Gago F, et al. Identification of aldo-keto reductase AKR1B10 as a selective target for modification and inhibition by prostaglandin $A(1)$ : implications for antitumoral activity. Cancer Res. (2011) 71:4161-71. doi: 10.1158/0008-5472.CAN-10-3816

41. Linker RA, Lee DH, Ryan S, van Dam AM, Conrad R, Bista P, et al. Fumaric acid esters exert neuroprotective effects in neuroinflammation via activation of the Nrf2 antioxidant pathway. Brain (2011) 134:678-92. doi: 10.1093/brain/awq386

42. Matsunaga T, Endo S, Takemura M, Soda M, Yamamura K, Tajima $\mathrm{K}$, et al. Reduction of cytotoxic p-quinone metabolites of tertbutylhydroquinone by human aldo-keto reductase (AKR) 1B10. Drug Metab Pharmacokinet. (2012) 27:553-8. doi: 10.2133/dmpk.DMPK-12NT-012

43. Endo S, Hu D, Suyama M, Matsunaga T, Sugimoto K, Matsuya Y, et al. Synthesis and structure-activity relationship of 2-phenyliminochromene derivatives as inhibitors for aldo-keto reductase (AKR) $1 \mathrm{~B} 10$. Bioorg Med Chem. (2013) 21:6378-84. doi: 10.1016/j.bmc.2013. 08.059

44. Li H, Yang AL, Chung YT, Zhang W, Liao J, Yang GY. Sulindac inhibits pancreatic carcinogenesis in LSL-KrasG12D-LSL-Trp53R172H-Pdx1-Cre mice via suppressing aldo-keto reductase family 1B10 (AKR1B10). Carcinogenesis (2013) 34:2090-8. doi: 10.1093/carcin/bgt170

45. Cao Z, Zhou B, Chen X, Huang D, Zhang X, Wang Z, et al. Statil suppresses cancer cell growth and proliferation by the inhibition of tumor marker AKR1B10. Anticancer Drugs (2014) 25:930-7. doi: 10.1097/CAD.0000000000000121

46. Chen H, Fu J, Chen H, Hu Y, Soroka DN, Prigge JR, et al. Ginger compound [6]-shogaol and its cysteine-conjugated metabolite (M2) activate Nrf2 in colon epithelial cells in vitro and in vivo. Chem Res Toxicol. (2014) 27:1575-85. doi: $10.1021 / \mathrm{tx} 500211 \mathrm{x}$

47. Cousido-Siah A, Ruiz FX, Mitschler A, Porté S, de Lera ÁR, Martín MJ, et al. Identification of a novel polyfluorinated compound as a lead to inhibit the human enzymes aldose reductase and AKR1B10: structure determination of both ternary complexes and implications for drug design. Acta Crystallogr D Biol Crystallogr. (2014) 70:889-903. doi: 10.1107/S139900471 3033452

48. Parsai S, Keck R, Skrzypczak-Jankun E, Jankun J. Analysis of the anticancer activity of curcuminoids, thiotryptophan and 4-phenoxyphenol derivatives. Oncol Lett. (2014) 7:17-22. doi: 10.3892/ol.2013.1679

49. Nishinaka T, Miura T, Sakou M, Hidaka C, Sasaoka C, Okamura A, et al. Down-regulation of aldo-keto reductase AKR1B10 gene expression by a phorbol ester via the ERK/c-Jun signaling pathway. Chem Biol Interact. (2015) 234:274-81. doi: 10.1016/j.cbi.2014.11.013

50. Zemanova L, Hofman J, Novotna E, Musilek K, Lundova T, Havrankova $\mathrm{J}$, et al. Flavones inhibit the activity of AKR1B10, a promising therapeutic target for cancer treatment. J Nat Prod. (2015) 78:2666-74. doi: 10.1021 /acs.jnatprod.5b00616

Conflict of Interest Statement: The authors declare that the research was conducted in the absence of any commercial or financial relationships that could be construed as a potential conflict of interest.

Copyright (c) 2018 Soares, Fachin, Trombone, Rosa, Ghidella and Belone. This is an open-access article distributed under the terms of the Creative Commons Attribution License (CC BY). The use, distribution or reproduction in other forums is permitted, provided the original author(s) and the copyright owner(s) are credited and that the original publication in this journal is cited, in accordance with accepted academic practice. No use, distribution or reproduction is permitted which does not comply with these terms. 